\title{
Analytical Solution of Nonprismatic Reinforced Concrete Cantilever Deflection under Concentrated Load
}

\author{
Amin Almasri \\ Civil Engineering Department, Fahad Bin Sultan University \\ Tabuk, Saudi Arabia \\ Jordan University of Science and Technology \\ Irbid, Jordan \\ aalmasri@fbsu.edu.sa; ahalmasri@just.edu.jo
}

\section{Extended Abstract}

Reinforced concrete cantilever beams are widely used in various types of applications. Cantilevers with varying depth along the length of the beam are widely used due to its aesthetic purposes, or due to saving some materials. However, deflections for such beams are usually obtained approximately using numerical techniques, like finite difference and finite elements. Obtaining an analytical solution for reinforced concrete beams with varying section can be tedious due to the fact that part of the concrete beam section will be cracked. Hence, an effective moment of inertia needs to be used instead of the gross moment of inertia to resist the deflection. In this research, an analytical solution is obtained for nonprismatic cantilever beam that has a varying depth along the length subjected to concentrated load at its end. The dependence of the solution on steel reinforcement ratio is simplified in order to obtain the solution. An effective moment of inertia model with tension stiffening is used in the formulation to obtain better prediction for deflection. It was found that the deflection is affected highly by the reinforcement ratio, the material modular ratio, in addition to the variation of the cross sectional area along the length. The obtained equation of deflection is easy and practical to be used by designers and engineers. 